\title{
OPTIMIZATION OF LONGITUDINAL AND LATERAL MOTION OF VEHICLE NEAR DISTURBED SURFACE
}

\author{
Alexander Knyazhsky \\ Department of Aerospace Measuring \\ and Computing Systems \\ Saint Petersburg State University of \\ Aerospace Instrumentation \\ Russia \\ knjagskij@mail.ru \\ Vladimir Nebylov \\ Alexander Nebylov \\ International Institute for \\ Advanced Aerospace Technologies \\ Russia \\ iiaat@aanet.ru \\ International Institute for \\ Advanced Aerospace Technologies \\ Russia \\ iiaat@aanet.ru
}

\section{Article history:}

Received 29.06.2018, Accepted 03.09.2018

\begin{abstract}
The paper covers the tasks of flight control, where it is necessary to minimize the mean true (geometrical) altitude of low-altitude vehicle flight. Therein a developed control algorithm for longitudinal and lateral motion of vehicle is described, assessment criteria for trajectory selection efficiency are determined and efficiency dependencies upon parameters of control algorithm and medium are analyzed.
\end{abstract}

\section{Key words}

Flight control, sensors of flight parameters, navigation, marine undispacement vehicle, wave disturbances, trajectory optimization.

\section{Introduction}

At the present time the important problem is the quality improvement for low-altitude vehicle motion control near underlying surface (Nebylov and Watson, 2016). Features of low-altitude motion are considered in the following works (Antonenko, Kitaev, Novikov, Bugaev and Zinevich, 2016; Rozhdestvensky, 2006). The interesting tasks exist where it is necessary to minimize the mean altitude of low-altitude vehicle motion trajectory. But if such criterion of vehicle trajectory selection is taken, the probability of vehicle touching the surface increases, and additional maneuvering increases the route to the final point. Some vehicles allow motion near the water surface. WIG-crafts, lowaltitude vehicles and helicopters, unmanned aircrafts, hydrofoil-supported crafts, air-cushion vehicles are among them (Yun, Bliault and Doo, 2010). During the long distance flight over the disturbed sea surface the probability of collision with the wave crests increases and it may cause accident (Pagowski and Szafran, 2014). Flying over the high waves increases flight motion safety and allows increasing maximum allowed altitude for flight (Pagowski, Szafran and Konczak, 2014).

Some structures of maritime low-altitude vehicle, namely, ground-effect craft, endure rare touching of water, but at their higher frequency and force the body can be damaged. Each touching affects the quality of navigation measurements, because vehicle altitude is changing at random basis and its flexible mode occurs. Considering all said above we can draw a conclusion that optimization of longitudinal and lateral-directional motion of low-altitude vehicle can be done by the criterion of mean geometrical height minimum of the flight at certain threshold of low probability of low-altitude vehicle touching the surface (Knyazhsky, Nebylov and Nebylov, 2017). According to some reasons there are restrictions for the values of occurring overloads, which shall be considered at selection of low-altitude vehicle control law.

It makes sense to review the waved sea surface of two types: solid (ground) and water (maritime). Correlation-extreme methods are used during the flight over the ground surface at minimum altitude in the mode of relief following to reduce radar signature or 
during monitoring of ground surface by optical and electronic instruments with bad resolution mounted at unmanned vehicle to forecast the relief of the ground and determine the most dangerous areas. But they can not be applied at motion over the wavy water surface that represents three-dimensional non-stationary stochastic field. That is why when flying over the sea it is necessary to forecast and partially pass around the long-period waves on the basis of obtained measurements of surface slope gradient under the vehicle by the corresponding position transducers. As far as rare short contacts of vehicle body with water are allowed during movement near the water surface, there is the possibility of lower-altitude flight, than flying over the ground surface. Ground-effect crafts use such flight at minimum altitude, where shielding effect is well shown, to improve aerodynamic efficiency and corresponding fuel economy (Knyazhskiy, Nebylov and Nebylov, 2017; Knyazhsky, Nebylov and Nebylov, 2017).

Range of Wing-in-Ground effect action depends on the vehicle size and sea conditions and usually includes altitudes from 1 to 10 meters. Specific motion altitude is set and stabilized as minimum allowed altitude from given low probability of wave crest touching.

During the flight over the ground surface for area monitoring in case of mean vehicle altitude minimization the probability of target detection increases.

Cost factors in many cases have critical importance during transport vehicle of one or another kind application tactics selection. Adding 3D motion control mode of low-altitude vehicle based on the supposed algorithm may be useful, practically have no influence on the development cost, but provides saving money at the vehicle exploitation.

\section{Mathematical Description of Low-altitude Vehi- cle Control System}

The main task of low-altitude vehicle automatic control consists in determination of different rudders deviations in current flight condition. Motion of lowaltitude vehicle as solid object in body axis coordinate system is described by the Euler equation. Forces and time periods appeared in the equation in complicated way depends upon altitude, velocity and flight mode and change in time with flight condition, for example, vehicle mass and moments of inertia. The task of low-altitude vehicle control algorithm development could be simplified by the review of two independently motion types: longitudinal and lateral.

Longitudinal motion can be described with the equations

$$
\left\{\begin{array}{l}
m \frac{d v}{d t}=P \cos \alpha-X_{a}-m g \sin \theta \\
m v \dot{\theta}=Y_{\alpha}+P \sin \alpha-m g \cos \theta \\
l_{z} \dot{\omega}_{z}=M_{z}
\end{array}\right.
$$

where $m$ is vehicle mass, $g$ is gravity acceleration, $v$ is linear velocity of vehicle mass center, $P$ is tractive force, $\alpha$ is attack angle , $\theta$ is angle of center mass motion trajectory climb, $X_{\alpha}$ is drag component, vector directed opposite to $\mathrm{V}$ vector, $Y_{a}$ is lifting force, vector perpendicular to $\mathrm{V}$ vector, $l_{z}$ is vehicle mass moment of inertia relatively axis $O Z_{\alpha}, \omega_{z}$ is pitching angular velocity, $M_{z}$ is moment of aerodynamic forces.

$$
M_{z}=m_{z} b_{a} S \frac{p V^{2}}{2}
$$

where $m_{z}$ is moment coefficient, $b_{a}$ is wing chord, $\rho$ is density of air, $S$ is wings area.

$$
\begin{gathered}
X_{a}=C_{x a} S \frac{p V^{2}}{2}, \\
Y_{a}=C_{y a} S \frac{p V^{2}}{2},
\end{gathered}
$$

where $C_{x a}$ is angle-of-attack dependent flight velocity coefficient of drag, $C_{y a}$ is angle-of-attack dependent flight velocity lift force coefficient.

Motion equation relative axis $\mathrm{OX}$ are represented by expression

$$
l_{x} \dot{\omega}_{x}=M_{x}
$$

where $l_{x}$ is vehicle moment of inertia relative axis $O X$, $M_{x}=M_{x}\left(\omega_{x}, V, \delta_{e}, H, \beta\right)$ is aerodynamic rotational moment, $\delta$ is angle of aileron deflection. Motion equations for yaw channel can also be determined with the help of projections external forces and forces of inertia on the axis $O Z_{a}$, normal to flight trajectory, and also external and inertia moments relatively axis $O Y$, perpendicular to $X Z$ plane:

$$
\left\{\begin{array}{l}
m V \dot{\Psi}=Z+P \sin \beta \\
l_{y} \dot{\omega}_{y}=M_{y} \\
\omega_{y}=\dot{\psi} \\
\psi=\Psi+\beta
\end{array}\right.
$$

where $Z$ is crosswind force, $l_{y}$ is inertia moment relatively axis $O Y_{c}, \psi$ and $\beta$ is azimuth angle and 
gliding angle.

During change of attack angle, azimuth angle, gliding angle, vehicle velocity and vehicle acceleration with the help of control drivers and engine thrusts, it is necessary to control the vehicle mass center motion in accordance with the required control laws.

\section{Measurement of Altitude}

Time-of-flight PMD cameras can be used for measuring the range up to several points of water surface (WS) and as a result the surface gradient under the vehicle. PMD (Photonic Mixer Device) technology is based upon phase method of active location in infrared range (Piatti D., Rinaudo F., 2012). Method essence pertains to measuring the time spent for passing the distance between the camera and surface by modulated optical infrared emission. Back-scattered radiation is measured by the transducer of camera images (lightsensitive matrix), after that the correlation levels are evaluated and phase deviations between radiated and received signals are calculated, which are proportional to the ranges up to the points of beam contact with water surface. PMD-camera application makes sense, when moving near the ground surface, because in case of large ranges there is a problem of ambiguity peculiar for all phase methods. When measurements are processed with special algorithms the height matrix $H$ of $l x d$ size is formed, the elements of which are the distance to the measured surface in the point of beam falling depending upon the radiation angles. Due to the fact that all beams come from one point (camera), but fall to different points of the space, the image is distorted due to increase of phase shift, the further the beam falls from the point of perpendicular entry, drawn from the radiation point to the water surface, the more the phase is shifted. Then it is necessary to perform the straightening of the measured water surface image. The range up to WS under the low-altitude vehicle, altitude and gradient of the vehicle can also be determined with the help of three location sensors mounted on the wing ends and the nose of the vehicle.

\section{Mathematical Description of Low-altitude Con- trol Algorithm}

Let us designate the matrix of vehicle trajectory parameters as $\theta(t)$ dependent of time $t$

$$
\theta(t)=[x(t) y(t) h(t) \gamma(t) v(t)]^{T}
$$

where $x(t)$ and $y(t)$ is rectangular plane topocentric coordinates of vehicle; $h(t)$ is altitude of vehicle center of gravity relatively the wavy water surface. $\gamma(t)$ is true course of vehicle; $v(t)$ is ground speed of vehicle, $v(t)=\sqrt{\left(\frac{d x(t)}{d t}\right)^{2}+\left(\frac{d y(t)}{d t}\right)^{2}} \theta(0)=\theta_{0}$ is initial parameters of vehicle movement.

Our task is to determine such $\theta(t)$, so that the conditions (7) are met and the difference between final and desired condition does not exceed the allowable deviation.

$$
\left\{\begin{array}{l}
\int_{0}^{T} h(\theta) d t=\min \\
v_{\gamma \min } \leq \frac{d \gamma(\theta)}{d t} \leq v_{\gamma \max } \\
P(h(\theta) \leq \xi(\theta)) \leq P_{0}
\end{array}\right.
$$

$P_{0}$ is minimum allowable probability of vehicle contact with wave, $T$ time of vehicle motion termination. It is considered that at the beginning of vehicle motion $t=0$.

Formula (7) means that it is necessary to minimize the trajectory height with restrictions of vehicle maneuvering angular velocity; the probability of contact with water surface shall not exceed the allowable value. Adding of condition $P(h(\theta) \leq \xi(\theta)) \leq P_{0}$ is due to the increase of probability of contact with water surface at reduction of fight altitude. The task of $\theta(t)$ trajectory routing at set velocity consists in determination of course and height $h$ in each period of time $t$.

Vehicle course is determined by the direction of the least height gradient in sector $\left[\gamma_{\min }, \gamma_{\max }\right]$

$$
\gamma(t)=\min \nabla \xi(x, y), \text { at } \gamma_{\min }(t)<\gamma(t)<\gamma_{\max }(t),
$$

Sector $\left[\gamma_{\min }, \gamma_{\max }\right]$ depends upon velocity, distance to designation point, direction of movement and waves' height, and has the center directed to the designation point. If $P_{0}$ is low, to reduce the probability of contact with water surface, if threshold value changing $\xi$ is exceeded by the velocity, the temporary increase of sector $\left[\gamma_{\min }, \gamma_{\max }\right]$ to pass around the wave crest is allowed.

\section{Simulation}

The process of simulation consists of 3 stages:

1. Simulation of developed wind-induced water waves of different grades;

2. Simulation of vehicle flight controlled by the developed algorithm;

3. Assessment of dependence of effective selection of optimized trajectory of vehicle motion upon the height of water waves, vehicle flight velocity, at restrictions of course changing speed.

Simulation of wind-induced water surface is done as per V. Pirson formula (Boroday I.K., Netsvetaev Yu.A., 1982). In this formula the wind-induced waves 
are represented as the superposition of a quantity of elementary two-dimensional cylindrical waves with different amplitudes, frequencies, phases and directions of distributions.

$$
\begin{gathered}
\xi(x, y)=\sum_{i=1}^{n} \sum_{j=1}^{m} r_{i j} \cos \left(k_{i} x \cos \left(\alpha_{j}\right)+\right. \\
\left.+k_{i} y \sin \left(\alpha_{j}\right)+\epsilon_{i j}\right)
\end{gathered}
$$

where $\xi(x, y)$ is wave height depending upon the spatial coordinates, $n$ is number of harmonics with different frequencies, $m$ is number of harmonic waves with different directions of spreading, $k_{i}$ is wave spatial frequency, $\alpha_{j}$ is angle that characterizes the direction of harmonic wave spreading, $r_{i j}$ is amplitude of wave with $i$-th frequency and $j$-th direction of spreading, $\epsilon_{i j}$ is phase represented by random number with uniform spreading. Spatial frequencies are determined by the formula $[1,2]$

$$
k_{i}=\Omega_{i}^{2} / g
$$

where $\Omega$ is root-mean-square frequency of spectrum.

$$
\Omega=0.77\left(\frac{g}{h_{3 \%}}\right)^{\frac{1}{2}}
$$

where $h_{3 \%}$ is height of water waves with provision of $3 \%$. In perfect condition $n$ and $m$ shall be very high values, but in (Nebylov, Alexander and Joseph Watson, 1982) it is shown that it is sufficient to take $n=m=7$ to simulate the developed non-regular wind-induced waves with high accuracy.

In Figure 1 the dotted line indicates the realization of trajectory of vehicle movement controlled by the described algorithm near the water surface with waves of 6 grades. The solid line shows the shortest trajectory of flight (if moving along the straight line).

Figure 2 shows the corresponding geometrical heights relatively the water surface.

1. Assessment of efficiency of automatic control algorithm

The criteria of flight control efficiency assessment shall consider the reduction of mean low-altitude vehicle height, wave height and probability of contact with the surface. In this work two efficiency criteria were introduced. The first one physically meant the reduction of man geometrical altitude of low-altitude vehicle flight relatively the waved sea surface under it. The second criterion evaluated the reduction of altitude of lowaltitude vehicle flight relatively the average sea level,

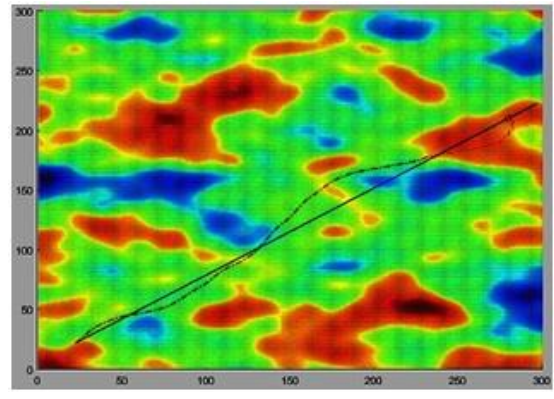

Figure 1. Realization of vehicle controlled movement trajectory near the water surface.

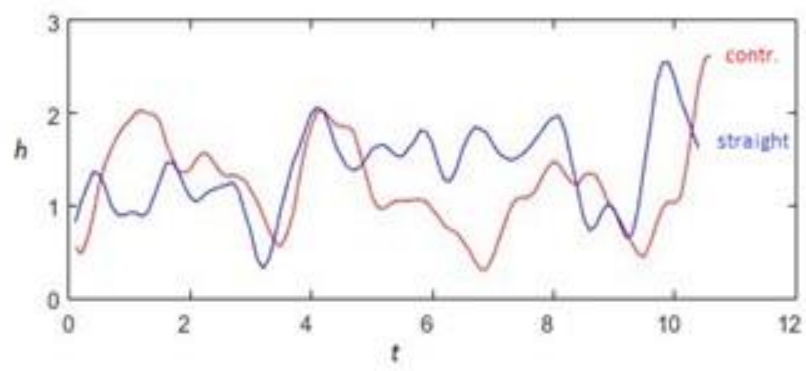

Figure 2. Dependence of vehicle altitude relatively waved sea surface under it upon the flight time.

i.e. absolute height. With the help of this criterion it is possible to forecast the reduction of radar and optical signature of low-altitude vehicle at control performed by the reviewed algorithm.

The dependence of efficiency assessment as per the first criterion upon the waves' power, vertical and horizontal velocities and restrictions for angular velocity was estimated by the formula:

$$
\begin{aligned}
& \chi\left(h_{3 \%}, v, \Delta \alpha_{\text {max }}, \Delta h_{\max }\right) \\
& \frac{\int_{0}^{T_{\text {uncontr }}} h\left(\theta_{\text {uncontr }}-\xi\left(\theta_{\text {uncontr }}\right)\right) d t}{l\left(\theta_{\text {uncontr }}\right)} \div \\
& \div \frac{\int_{0}^{T_{\text {contr }}}\left(h\left(\theta_{\text {contr }}\right)-\xi\left(\theta_{\text {contr }}\right)\right) d t}{l\left(\theta_{\text {contr }}\right)}= \\
& =\frac{l\left(\theta_{\text {contr }}\right) \int_{0}^{T_{\text {uncontr }}} h\left(\theta_{\text {uncontr }}-\xi\left(\theta_{\text {uncontr }}\right)\right) d t}{l\left(\theta_{\text {uncontr }}\right) \int_{0}^{T_{\text {contr }}}\left(h\left(\theta_{\text {contr }}\right)-\xi\left(\theta_{\text {contr }}\right)\right) d t}
\end{aligned}
$$

at probability of contact with water surface less than $P_{0}$, where $\theta_{\text {contr }}$ is trajectory parameters at controlled motion, $\theta_{\text {uncontr }}$ is trajectory parameters at movement along the shortest distance, $\xi(\theta)$ is height of waves under $\theta$ trajectory, $l(\theta)$ is length of $\theta$ trajectory, $\Delta \alpha_{\max }$ is maximum speed of course changing, $\Delta h_{\max }$ is maximum speed of height changing, $T_{\text {uncontr }}-$ arrival time to designation point at flying along the shortest (straight) trajectory, $T_{\text {contr }}$ - arrival time to designation point at flying along the controlled 
trajectory.

Formula (12) physically means the ratio of mean true altitude of low-altitude vehicle during the flight along the shortest trajectory to mean true altitude at flight as per the controlled trajectory.

Efficiency dependence upon medium parameters and maneuvering characteristics of vehicle can be presented by multi-dimensional surface $\chi$ in orthogonal basis $\left.\left(\bar{\chi}, \overline{h_{3 \%}}\right), \bar{v}, \Delta \bar{\alpha}_{\max }, \Delta \bar{h}_{\max }\right)$. For the assessment of algorithm quality it makes sense to calculate the mean efficiency. The mean value of surface in multi-dimensional space that has no negative values is obtained as the volume of figure under the surface divided by the product of its lengths by basis axes.

In the result of series of experiments there were obtained the averaged dependencies of efficiency upon vehicle velocities and wave height.

Figure 3 shows the dependency of efficiency, evaluated as per the first criterion, upon vehicle speed at $h_{3 \%}=6$ $\mathrm{m}$, obtained by mathematical simulation.

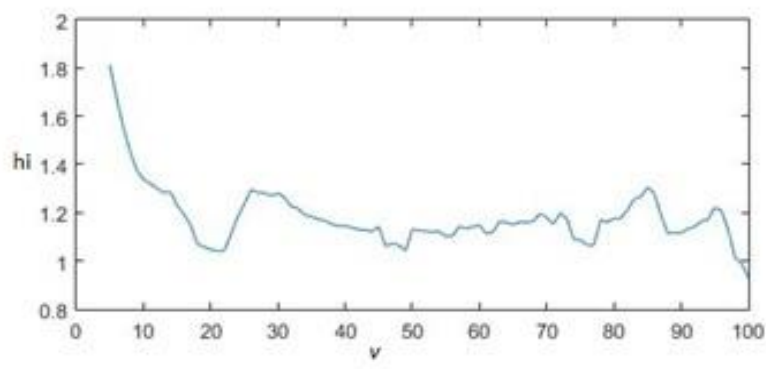

Figure 3. Dependency of efficiency, evaluated as per the first criterion, upon vehicle speed

When the speed is increased, the flight mean altitude relatively the water surface is increased to maintain specified $P_{0}$, because vehicle has no time to pass around all elevations. Figure 4 shows the dependency of efficiency upon wave height at $v=33 \mathrm{~m} / \mathrm{s}$, obtained by simulation.

When wave height increases at fixed speed, control efficiency is also increased, because wave length is increased and probability of vehicle getting into the hollow place is increased at proper control. Mean efficiency of vehicle control as per the described algorithm is equal to 1.129 if assessed by the first criterion. The dependence of efficiency assessment as per the second criterion upon the waves' power, vertical and horizontal velocities and restrictions for angular velocity was estimated by the formula:

$$
\chi\left(h_{3 \%}, v, \Delta \alpha_{\max }, \Delta h_{\max }\right)
$$

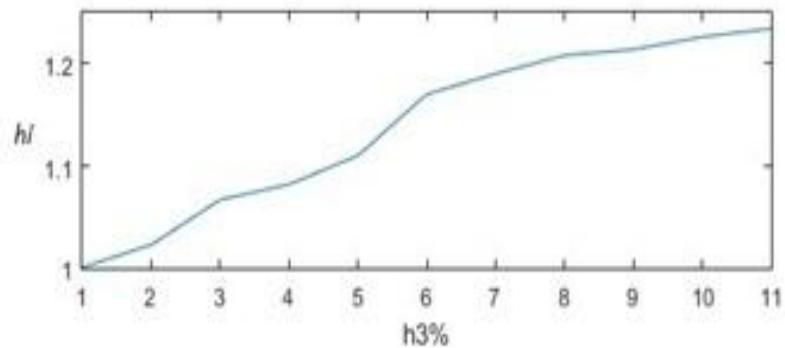

Figure 4. dependency of efficiency, evaluated as per the first criterion, upon wave height

$$
\begin{gathered}
\frac{\int_{0}^{T_{\text {uncontr }}} h\left(\theta_{\text {uncontr }}\right) d t}{l\left(\theta_{\text {uncontr }}\right)} \div \frac{\int_{0}^{T_{\text {contr }}} h\left(\theta_{\text {contr }}\right) d t}{l\left(\theta_{\text {contr }}\right)}= \\
=\frac{l\left(\theta_{\text {contr }}\right) \int_{0}^{T_{\text {uncontr }}} h\left(\theta_{\text {uncontr }}\right) d t}{l\left(\theta_{\text {uncontr }}\right) \int_{0}^{T_{\text {contr }}} h\left(\theta_{\text {contr }} d t\right.}
\end{gathered}
$$

at probability of contact with water surface less than $P_{0}$, where $\theta_{\text {contr }}$ - trajectory parameters at controlled motion, $\theta_{\text {uncontr }}$ - trajectory parameters at movement along the shortest distance, $\xi(\theta)$ - height of waves un$\operatorname{der} \theta$ trajectory, $l(\theta)$ - length of $\theta$ trajectory, $\Delta \alpha_{\max }$ - maximum speed of course changing, $\Delta h_{\max }$ maximum speed of height changing, $T_{\text {uncontr }}-$ arrival time to designation point at flying along the shortest trajectory, $T_{\text {contr }}$ - arrival time to designation point at flying along the controlled trajectory. For the assessment of general efficiency of movement optimization as per the second criterion it is necessary to integrate $\chi$ as per $h_{3 \%}, v, \Delta \alpha_{\max }, \Delta h_{\max }$. Figure 5 shows the dependency of efficiency, evaluated as per the second criterion, upon vehicle velocity at $h_{3 \%}=6$ $\mathrm{m}$, obtained by simulation.

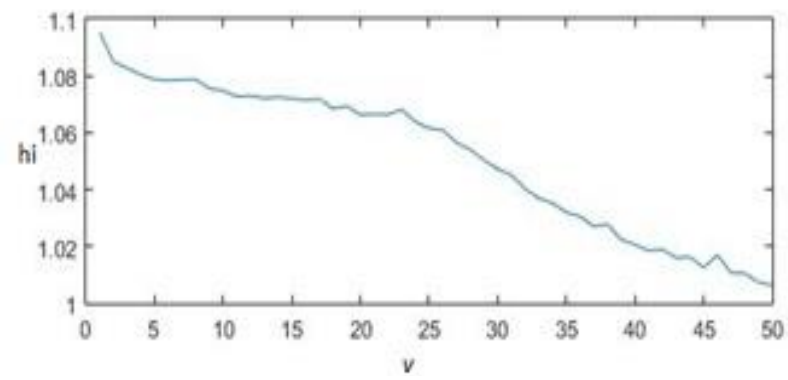

Figure 5. Dependency of efficiency, evaluated as per the second criterion, upon vehicle velocity in $\mathrm{m} / \mathrm{s}$.

When the speed is increased, the efficiency evaluated as per the second criterion decreases and has lower values than at evaluation as per the first criterion. Figure 
6 shows the dependency of efficiency evaluated as per the second criterion upon wave height at $v=33 \mathrm{~m} / \mathrm{s}$, obtained by simulation.

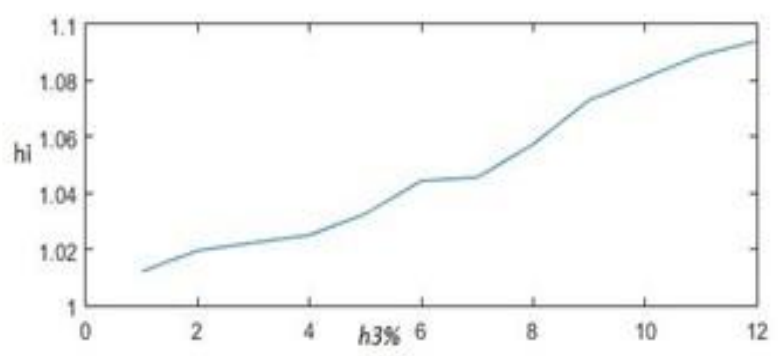

Figure 6. Dependency of efficiency, evaluated as per the second criterion, upon wave height

When wave height increases, the efficiency, evaluated as per the second criterion, also increases, but with lower speed than at evaluation as per the first criterion. The example of the first criterion use can be the task of evaluating the efficiency of ground-effect craft lifting force increase, which directly depends upon its proximity to waved sea surface to demonstrate ground effect. One of the tasks with the use of the second criterion at flight over the ground surface is monitoring of forest fires with the application of optical and electronic means of low resolution.

\section{Conclusion}

In the result of simulation it was stated that the described algorithm of vehicle control allows reducing flight altitude relatively wind-induced waved sea surface. The dependency of low-altitude vehicle control efficiency upon its speed and wave height at movement near water surface was evaluated. General efficiency of algorithm was evaluated when it is used within speed range of $1-100 \mathrm{~m} / \mathrm{s}$ and wave height with $3 \%$ provision in 1-11 meters. The obtained results show that when low-altitude vehicle velocity is increased at fixed wave height, the efficiency of control algorithm reduces, when wave height increases with fixed speed, the efficiency of control algorithm increases. The obtained results mean that it is reasonable to use the above algorithm of vehicle control to minimize the mean geometrical altitude of flight at relatively small velocities for not civil low-altitude vehicles. The paper innovation idea consists in adding the secondary mode of automatic control for low-altitude flight vehicle designed for passing over high wave crests. The control principle requires finding of minimum altitude gradient in definite sector.

\section{Acknowledgements}

The work was supported by the Russian Science Foundation under the project 16-19-10381 and by the Russian Foundation for Basic Research under the project 15-08-00423-a

\section{References}

Antonenko S.V., Kitaev M.V., Novikov V.V., Bugaev V.G., and Zinevich A.N. (2016). The research of an airfoil boat take-off stage, 26th annual international ocean and polar engineering conference, ISOPE 2016, June 26-July 01 2016, Greece, pp. 808813.

Boroday I.K. and Netsvetaev Yu.A. (1982). Navigability of vessels. L.: Shipbuilding.(in Russian).

Knyazhskiy A.Y., Nebylov A.V., and Nebylov V.A. (2017). Optimization of WIG-craft 3D-trajectory near the rough sea surface, EUCASS (European Conference for AeroSpace Sciences), 3-6 July 2017, Milan, Italy.

Knyazhskiy A.Y., Nebylov A.V, and Nebylov V.A. (2017). Methods for Signal Processing and Motion Control of Ground Effect Vehicle. 4th IEEE International Workshop on Metrology for Aerospace. Padua, Italy, June 21-23, 2017.

Knyazhsky A.Yu., Nebylov A.V., Nebylov V.A. (2017). Increase in the aerodynamic quality of ground effect vehicle due to the big waves turning around. Cybernetics and Physics. 6(2), pp. 71-75.

Nebylov, Alexander and Joseph Watson, Editors. (2016). Aerospace Navigation Systems. John Wiley \& Sons, Ltd, UK.

Pagowski Z.T., Szafran K. (2014). "Ground effect" Inter-Modal Fast Sea Transport. TransNav the International Journal on Marine Navigation and Safety of Sea Transportation, Warsaw, Poland, 8(2), pp.317320.

Pagowski Z.T., and Szafran K.,Konczak J.(2014) "Ground effect" Transport on the Baltic Sea, Maritime Transport \& Shiping Marine Navigation and Safety of Sea Transportation, Warsaw, Poland, 8(2), pp.221-234.

Piatti D., and Rinaudo F. (2012). SR-4000 and CamCube3.0 Time of light (ToF) Cameras: Tests and Comparison. Remote Sensing, 1069-1089.

Rozhdestvensky K.V. (2006). Wing-in-ground effect vehicles. Progress in Aerospace Sciences, 42(3), pp. 211-283.

Yun L. , Bliault A., and Doo J. (2010). WIG Craft and Ekranoplan: Ground Effect Craft Technology. Springer. 